\title{
The mevalonate pathway but not the methylerythritol phosphate pathway is critical for elaioplast and pollen coat development in Arabidopsis
}

\author{
Keiko Kobayashi', Masashi Suzuki ${ }^{2,3}$, Toshiya Muranaka ${ }^{2,4}$, Noriko Nagata, ${ }^{1, *}$ \\ ${ }^{1}$ Department of Chemical and Biological Sciences, Faculty of Science, Japan Women's University, 2-8-1 Mejirodai, Bunkyo- \\ ku, Tokyo 112-8681, Japan; ${ }^{2}$ Plant Science Center, Riken, 1-7-22 Suehiro-cho, Tsurumi-ku, Yokohama, Kanagawa 230-0045, \\ Japan; ${ }^{3}$ The Chemical Biology Laboratory, Graduate School of Agricultural and Life Sciences, The University of Tokyo, \\ 1-1-1 Yayoi, Bunkyo-ku, Tokyo 113-8657, Japan; ${ }^{4}$ Department of Biotechnology, Graduation School of Engineering, Osaka \\ University, 2-1 Yamadaoka, Suita, Osaka 565-0871, Japan \\ *E-mail: n-nagata@fc.jwu.ac.jp Tel \& Fax: +81-3-5981-3663
}

Received April 12, 2018; accepted July 2, 2018 (Edited by Y. Ito)

\begin{abstract}
Pollen coat components are derived from tapetum cells, which contain elaioplasts derived from plastids and tapetosome derived from the endoplasmic reticulum. In Brassica napus, the main neutral lipids in the elaioplast and tapetosome have been reported to be sterol ester and triacylglycerol, respectively. Isopentenyl pyrophosphate, the structural component of sterol, is produced via the cytosolic mevalonate (MVA) and plastidic methylerythritol phosphate (MEP) pathways. Although these two pathways are compartmentalized, partial cross-talk between them has been reported. To investigate the contribution of these two pathways in elaioplast formation, we characterized mutant pollen of these two pathways. We observed the anthers of male sterile hmg1-1 and atipi1 atipi2 mutants ultrastructurally, which were deficient in MVA pathway enzymes. hmg1-1 and atipilatipi2 showed a shrunken elaioplast inner granule at the bicellular pollen stage. Conversely, in the cla1-1 mutant, which showed a defective MEP pathway, elaioplast development was normal. The pollen of hmg1-1 and atipilatipi2 was coatless, whereas cla1-1 had a pollen coat. These results indicate that the MVA pathway but not the MEP pathway is critical for elaioplast development though the organelle is derived from plastids.
\end{abstract}

Key words: gametophyte, isoprenoid, sterol, tapetosome, tapetum.

A mature male gametophyte is covered by exine and a pollen coat. The main component of exine is a sporoporenine polymer that comprises lipids and phenolic derivatives. Exine guards pollen grains chemically and physically (Piffanelli et al. 1998). Conversely, the pollen coat mainly comprises lipids and protein and it has many properties, such as attaching to vector insects to facilitate pollen transmission (Piffanelli et al. 1998), attaching to the dry stigmatic surface to start pollen hydration (Hülskamp et al. 1995; Preuss et al. 1993), and self-incompatibility (Shiba et al. 2001).

The pollen coat is derived from tapetum cells at the innermost cell layer of the anther wall, which contains the elaioplast and tepetosome (Hernández-Pinzón et al. 1999). The elaioplast is an organelle derived from plastids, and the tapetosome is an organelle derived from the endoplasmic reticulum (ER). In Arabidopsis, these organelles develop in tapetum cells when a male gametophyte is in the bicellular pollen stage (Quilichini et al. 2014). In the tricellular pollen stage, tapetum cells disappear and a pollen coat forms at the microspore surface. It is considered that the pollen coat contains sterol ester as the major natural lipid and contains phospholipid as a polar lipid. Various sterol types exist in the pollen of different plant species (Villette et al. 2015). Lipid profiles of tapetum organelles are very different between the elaioplast and tapetosome. In a previous report, the major natural lipids of elaioplasts were identified as sterol ester and wax ester, while that of the tapetosome was triacylglycerol in Brassica napus (Hernández-Pinzón et al. 1999). Arabidopsis knock-down mutants of acetoacethyl CoA synthase (AACT2) (Jin et al. 2012) and HMG-CoA synthase (HMGS) (Ishiguro et al. 2010) also show a pollen coatless phenotype and male sterility. AACT2 and HMGS are biosynthetic enzymes up-stream of sterol biosynthesis.

Sterol is a kind of isoprenoid. Isoprenoid compounds comprise isopentenyl diphosphate (IPP)

Abbreviations: AACT, acetoacethyl CoA synthase; DMAPP, dimethylallyl pyrophosphate; ER, endoplasmic reticulum; HMGR, 3-hydroxy-3methylglutaryl-CoA reductase; HMGS, HMG-CoA synthase; IPI, isopentenyl pyrophosphate isomerase; IPP, isopentenyl pyrophosphate; MEP, methylerythritol phosphate; MS, Murashige and Skoog; MVA, mevalonate; WT, wild type.

This article can be found at http://www.jspcmb.jp/

Published online October 25, 2018 


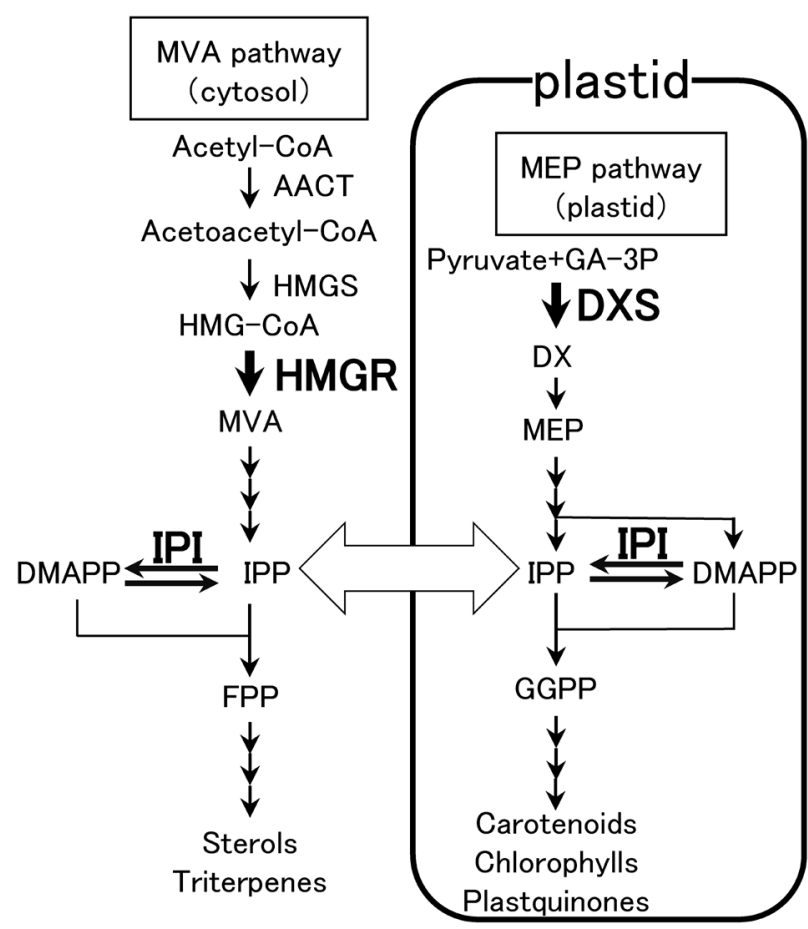

Figure 1. Scheme of the isoprenoid biosynthesis pathway. AACT, Acetoacetyl CoA thiolase; HMGS, 3-hydroxy-3-methylglutaryl-CoA synthase; HMG-CoA, 3-hydroxy-3-methylglutaryl-CoA; HMGR, HMG-CoA reductase; IPP, isopentenyl diphosphate; DMAPP, dimethyl allyl diphosphate; IPI, Isopentenyl/dimethylallyl diphosphate isomerase; FPP, farnesyl diphosphate; GA-3P, Glyceraldehyde-3phosphate; DXS, 1-deoxy-D-xylulose 5-phosphate synthase; DX, 1-deoxy-D-xylulose 5-phosphate; MEP, methylerythritol phosphate; and GGPP, geranylgeranyl diphosphate. The white arrow signifies metabolite cross-talk between the cytosolic MVA pathway and plastidic MEP pathway.

and dimethylallyl diphosphate (DMAPP), which are synthesized via the cytosolic MVA pathway and the plastidic methylerythritol phosphate (MEP) pathway (Figure 1). These two pathways are separated by the plastid membrane (Lichtenthaler et al. 1997). Although there is partial metabolic cross-talk between MVA and MEP pathways, the mechanisms remain unclear (Kasahara et al. 2002; Nagata et al. 2002).

It is strange that sterol accumulates in elaioplasts which are derived from plastids (Hernández-Pinzón et al. 1999), although sterol is known as the cytosolic MVA pathway derivative (Newman and Chappell 1999).

In the $h m g s / f k p 1$ mutant, the structure of elaioplasts and the tapetosome is strange, formation of the pollen coat and pollen hydration is inhibited, and the fkp1 mutant exhibits male sterility (Ishiguro et al. 2010). These findings suggest that the sterol of elaioplasts is derived from the MVA pathway.

In a previous study, we isolated the male sterile mutant, which was a defective mutant of 3-hydroxy-3methylglutaryl-CoA reductase (HMGR), the key enzyme of the MVA pathway (Suzuki et al. 2004). In Arabidopsis, HMGR is encoded by two genes, HMG1 and HMG2.
In anthers, HMG1 was expressed in the tapetum and microspores, while the expression of HMG2 appeared only in microspores (Suzuki et al. 2009). The hmg1-1 mutant showed an inhibition of pollen germination or pollen tube elongation (Suzuki et al. 2004). Besides the RNAi mutant of AACT2, hmgs/fkp1 mutant, and hmg1 mutant (Ishiguro et al. 2010; Jin et al. 2012; Suzuki et al. 2004), the atipilatipi2 mutant (Okada et al. 2008) has been isolated as a male sterile mutant, which is defective in the MVA pathway. Isopentenyl pyrophosphate isomerase (IPI) catalyzes the isomerization reaction from IPP to DMAPP. Two genes encoding IPI, IPI1 and IPI2, exist in the Arabidopsis genome. DMAPP is necessary for isoprenoid biosynthesis as an enzyme substrate, such as farnesyl diphosphate synthase and geranylgeranyl diphosphate synthase.

cla1-1 is a null mutant of the first enzyme of the MEP pathway, 1-deoxy-D-xylulose-5-phosphate synthase 1 (Estévez et al. 2000). In this mutant, the accumulation of chlorophyll and carotenoids is totally inhibited in the chloroplast and this mutant shows an albino phenotype (Mandel et al. 1996). In this study, we investigated the role of the MEP pathway on elaioplast development using Arabidopsis flowers of cla1-1. We observed the developmental stage of microspores in these mutants and demonstrated the contribution of the MVA pathway but not the MEP pathway on elaioplast development.

We observed and compared the ultrastructure of anthers between wild type (WT) and male sterile mutants of the MVA pathway, hmg1-1 and atipilatipi2. In the bicellular pollen stage, electron-dense granules in the elaioplast were shrunken in $h m g 1-1$ and atipilatipi2 (Figure 2A-H). The area of high electron density in this experimental condition was generally considered a lipid-rich area. Thus, these results showed that lipids in the elaioplast were decreased in hmg1-1 and atipilatipi2. The tapetosome in hmg1-1 and atipilatipi2 was smaller than that in WT (Figure 2A-H). In the late tricellular pollen stage, a pollen coatless phenotype was observed in hmg1-1 and atipilatipi2 (Figure 2I-P). These results are consistent with previous results wherein mutants of the MVA pathway, the RNAi mutant of AACT2 (Jin et al. 2012) and $h m g s / f k p 1$ (Ishiguro et al. 2010), showed a pollen coatless phenotype. The elaioplasts in $h m g s / f k p 1$ mutant, $h m g 1-1$ mutant and atipilatipi2 mutant showed an abnormal shape, although the genes encoding plastidic isoprenoid biosynthetic enzymes are not affected in these mutants (Figure $2 \mathrm{~A}-\mathrm{H}$ ). A complete blockage of the MVA pathway resulted in a lethal phenotype (Ishiguro et al. 2010; Jin et al. 2012; Suzuki et al. 2009). Another allele of atipilatipi2, idi1idi2, has been reported to be lethal (Phillips et al. 2008). Although the molecular mechanism of the difference is unclear, differences in the genetic lines between them may cause this phenotypic variation. atipilatipi2 was 


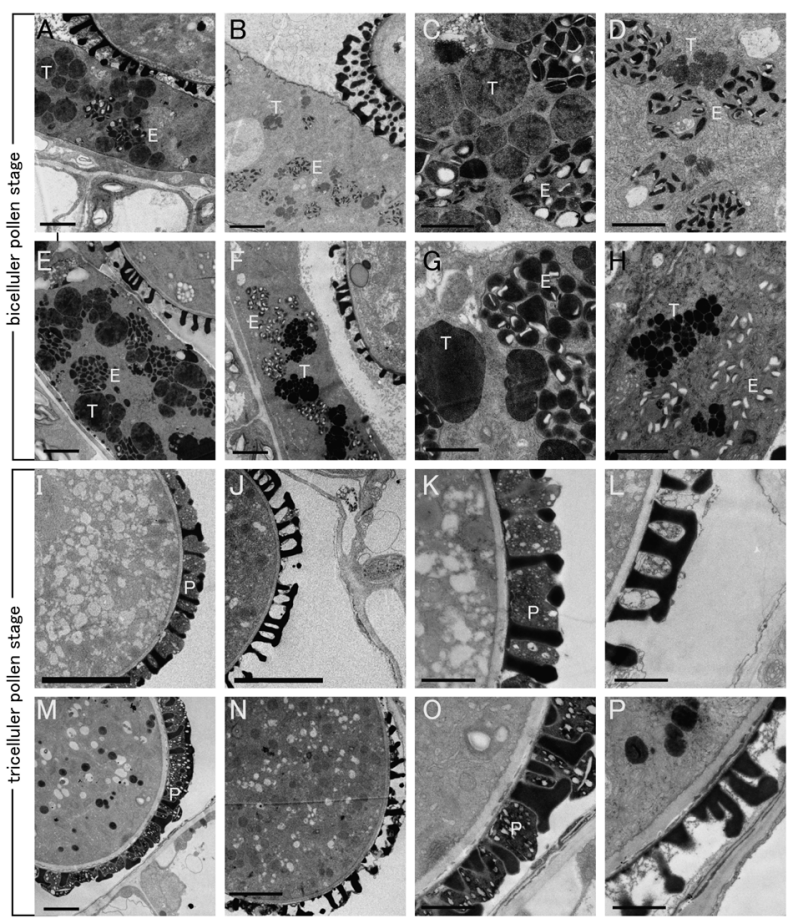

Figure 2. Abnormal elaioplast and pollen coat of hmg1-1 and atipilatipi2. The developmental stage of a male gametophyte in WT (WS; A, C, I, K Col-0; E, G, M, O), hmg1-1 (B, D, J, L), and atipilatipi2 (F, H, N, P) mutants. Tapetal organelle development in the bicellular pollen stage $(\mathrm{A}-\mathrm{H})$. Pollen coat in the tricellular pollen stage (I-P). E, elaioplast; T, tapetosome; P, pollen coat. Scale bars $=2 \mu \mathrm{m}$ in A, B, E, F, and $\mathrm{m}, 1 \mu \mathrm{m}$ in $\mathrm{C}, \mathrm{D}, \mathrm{G}, \mathrm{H}, \mathrm{K}, \mathrm{L}, \mathrm{O}$, and $\mathrm{P}$, and $5 \mu \mathrm{m}$ in $\mathrm{I}$, J, and $\mathrm{N}$.

viable probably because 7\% WT IPI activity remained in atipilatipi2 (Okada et al. 2008). However, we demonstrated that 7\% WT IPI activity in atipilatipi2 may be insufficient for normal differentiation of the elaioplast and formation of the pollen coat. These results were also consistent with the results of $h m g s / f k p 1-1$ (Ishiguro et al. 2010).

The tapetosome also showed an abnormal phenotype in $h m g 1-1$, atipilatipi2 (Figure 2A-H), and $h m g s / f k p 1-1$. These results suggest that the cytosolic MVA pathway contributed to the formation of a pollen coat, elaioplast, and tapetosome. A sterol-deficient mutant, such as defective mutants of the MVA pathway, may also show a pollen coatless phenotype because sterol ester, the main component of a pollen coat, was decreased.

The next question is the importance of the products from the MEP pathway in the elaioplast. To investigate the contribution of MEP pathway to elaioplast development, we performed an electron microscopic analysis of the microspore in cla1-1. Most cla1-1 homozygous mutants showed a lethal seedling phenotype when grown on Murashige and Skoog (MS) agar. However, when the seedlings were transferred to $1 / 2 \mathrm{MS}$ agar supplemented with $1.5 \%$ sucrose in a large agripot, only some that grew to the flower stage were obtained (Figure 3A-D). Mature cla1-1 plants showed a sterile
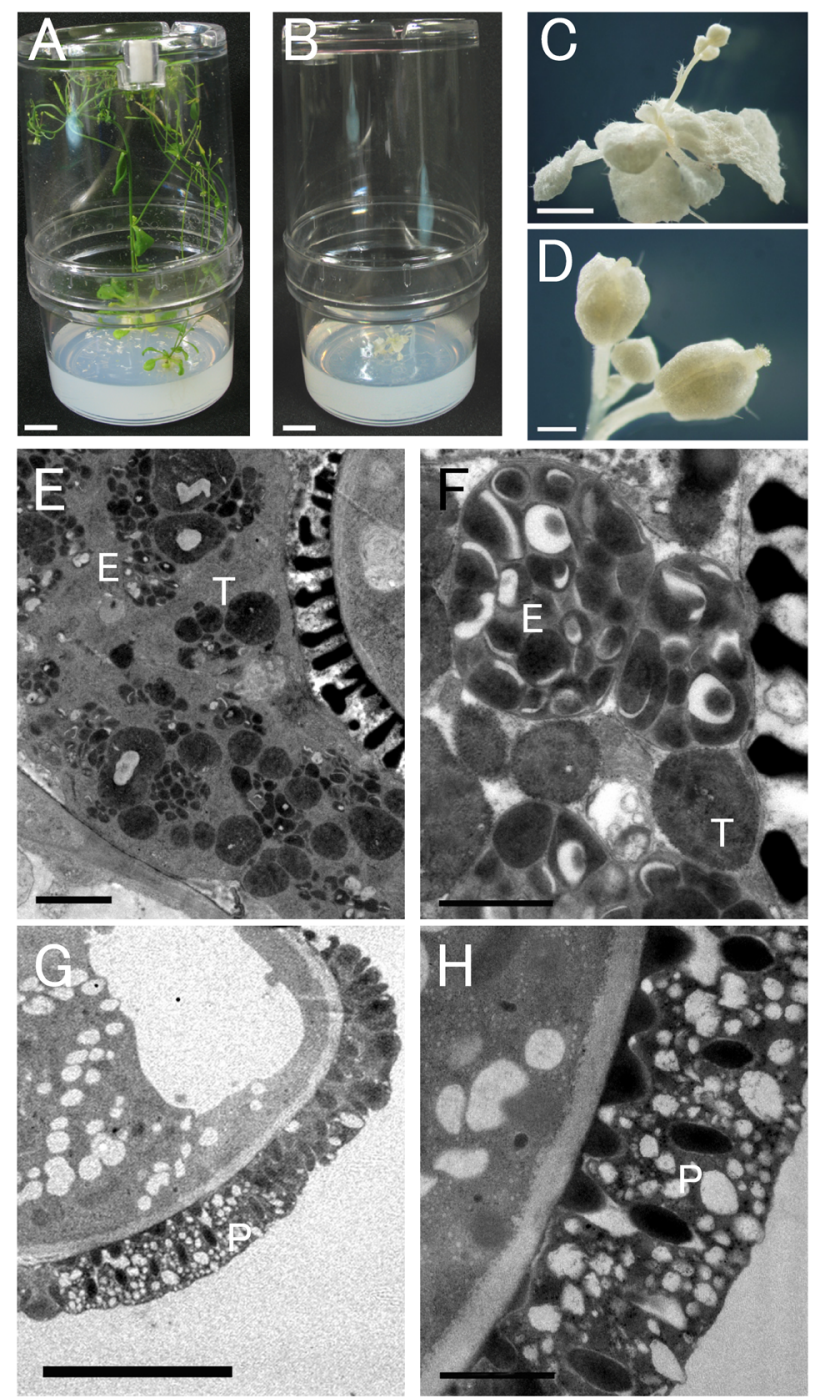

Figure 3. Normal elaioplast and pollen coat of cla1-1. A 40-dayold WT (Ws; A) and cla1-1 (B) plant. Close-up view of the plant (C) and flower (D) of cla1-1. Tapetal organelle development of cla1-1 in the bicellular pollen stage (E, F). Pollen coat of cla1-1 in the tricellular pollen stage $(\mathrm{G}, \mathrm{H})$. P, pollen coat. The abnormal vacuole is indicated by an asterisk. Scale bars $=1 \mathrm{~cm}$ in A and B, $2 \mathrm{~mm}$ in C, $400 \mu \mathrm{m}$ in D, $2 \mu \mathrm{m}$ in $\mathrm{E}, 1 \mu \mathrm{m}$ in $\mathrm{F}, \mathrm{H}$, and $5 \mu \mathrm{m}$ in $\mathrm{G}, \mathrm{I}, \mathrm{J}$.

phenotype wherein the primary shoot terminated at some buds. At the bicellular pollen stage, the elaioplast and tapetosome of cla1-1 were comparable to that of WT (Figure 2A, C and Figure 3E, F). In the tricellular pollen stage, the pollen coat formed normally (Figure 2I, K and Figure $3 \mathrm{G}, \mathrm{H}$ ), although almost all gametophytes had abnormal vacuoles (Figure 3G) or were crushed in cla1-1 (data not shown). At least the male gametophytes were indistinguishable between WT and cla1-1 at bicellular pollen stage, while normal development may be prevented thereafter in cla1-1 (Supplementary Figure). From these results, we considered that the sterile cla1-1 phenotype may have been caused by an abnormal gametophyte.

These results suggest that the MEP pathway, unlike the 
MVA pathway, was not necessary for the formation of the elaioplast or pollen coat. In the tricellular pollen stage, there were many abnormal gametophytes in the cla1-1 mutant. This result indicated that the cla1-1 mutant had no ability for normal pollen development after the tricellular pollen stage, suggesting that the MEP pathway is important in the formation of the gametophyte but not of the elaioplast.

It has been reported that sterol ester accumulates in the elaioplast in B. napus (Hernández-Pinzón et al. 1999). The abnormal elaioplast, pollen coatless microspores, and male sterility phenotypes were observed not only in $h m g s / f k p 1-1$ (Ishiguro et al. 2010) but also in both hmg1-1 and atipilatipi2 in this study. Our results strongly support the previous hypothesis that sterol ester in the elaioplast is derived from the cytosolic MVA pathway, even though the elaioplast is derived from plastids. Two possibilities have been proposed: (1) MVA and sterol biosynthesis pathways localize in a special organelle, which is the elaioplast and (2) sterols are produced in cytosol and then transported to the elaioplast. The possibility of (1) may be low as there are more than 11 steps in sterol synthesis from acetyl CoA (Suzuki and Muranaka 2007). Although it is necessary that enzymes with more than 11 synthesis steps are imported to the elaioplast, these 11-step enzymes do not have a plastidtransfer signal. On the contrary, it was reported that ER covered the proplastid in the process of formation of elaioplast from proplastid in Olea europaea (Pacini and Casadoro 1981). It is likely that sterols were transported to the elaioplast from ER. The trafficking of intermediary isoprenoid metabolites between plastids and cytosol has been reported (Hemmerlin et al. 2003; Kasahara et al. 2002; Nagata et al. 2002). Elucidation of the mechanisms how MVA pathway products accumulate in plastids may reveal the developmental mechanisms of the elaioplast in pollen growth stages.

\section{Acknowledgements}

We thank Eriko Suzuki and Rumi Aoyama for their contribution to the electron microscopic analysis. We thank Drs. Kazunori Okada and Patricia León for kindly providing ipi1-ipi2 double mutant and cla1-1 respectively. This work was supported by MEXT KAKENHI Grant Nos. 23570082 and 26440170 to NN.

\section{Appendix}

\section{Materials and methods}

\section{Plant growth condition}

Seeds were sown on 1/2 MS medium (Wako Pure Chemical Industries, Ltd., Japan) supplemented with $1.5 \%(\mathrm{w} / \mathrm{v})$ sucrose and stored at $4^{\circ} \mathrm{C}$ for more than 2 day. After vernalization, WT (WS, Col-0), hmg1-1 (Suzuki et al. 2004), and atipilatipi2 (Okada et al. 2008) plants were grown for two weeks on 1/2 MS agar medium and then transferred to soil. cla1-1 (Mandel et al. 1996) plants were grown for two weeks on 1/2 MS agar and then transferred to $1 / 2 \mathrm{MS}$ agar in an agripot. These plants were cultured for 40-50 day. All growth occurred under a $16: 8 \mathrm{~h}$ light: dark cycle at $23^{\circ} \mathrm{C}$ in a growth chamber.

\section{Electron microscopic analysis}

For transmission electron microscopy, the samples were fixed with $4 \%$ glutaraldehyde and $4 \%$ paraformaldehyde in a $20 \mathrm{mM}$ sodium cacodylate buffer, $\mathrm{pH} 7.0$, at $4^{\circ} \mathrm{C}$ overnight. They were then washed with the same buffer for $4 \mathrm{~h}$ at $4^{\circ} \mathrm{C}$. Then, they were post-fixed with $2 \% \mathrm{OsO}_{4}$ in a $20 \mathrm{mM}$ sodium cacodylate buffer at $4^{\circ} \mathrm{C}$ overnight. The fixed samples were run through an alcohol series and embedded in Spurr resin. Ultra-thin sections ( $70 \mathrm{~nm}$ thick) were cut with a diamond knife on an ULTRACUT E ultra-microtome (Leica, Wien, Austria) and transferred to Formvar-coated grids. They were doublestained with $4 \%(\mathrm{v} / \mathrm{v})$ uranyl acetate for $15 \mathrm{~min}$ and with lead citrate solution for $7 \mathrm{~min}$. After washing with distilled water, the samples were observed using a JEM-1200 EX transmission electron microscope (Jeol, Tokyo, Japan).

\section{References}

Estévez JM, Cantero A, Romero C, Kawaide H, Jiménez LF, Kuzuyama T, Seto H, Kamiya Y, Léon P (2000) Analysis of the expression of CLA1, a gene that encodes the 1-deoxyxylulose 5-phosphate synthase of the 2-C-methyl-D-erythritol-4phosphate pathway in Arabidopsis. Plant Physiol 124: 95-104

Hemmerlin A, Hoeffler JF, Meyer O, Tritsch D, Kagan IA, Grosdemange-Billiard C, Rohmer M, Bach TJ (2003) Crosstalk between the cytosolic mevalonate and the plastidial methylerythritol phosphate pathways in tobacco bright yellow-2 cells. J Biol Chem 278: 26666-26676

Hernández-Pinzón I, Ross JHE, Barnes KA, Damant AP, Murphy DJ (1999) Composition and role of tapetal lipid bodies in the biogenesis of the pollen coat of Brassica napus. Planta 208: 588-598

Hülskamp M, Kopczak SD, Horejsi TF, Kihl BK, Pruitt RE (1995) Identification of genes required for pollen-stigma recognition in Arabidopsis thaliana. Plant J 8: 703-714

Ishiguro S, Nishimori Y, Yamada M, Saito H, Suzuki T, Nakagawa T, Miyake H, Okada K, Nakamura K (2010) The arabidopsis FLAKY POLLEN1 gene encodes a 3-hydroxy-3-methylglutarylcoenzyme a synthase required for development of tapetumspecific organelles and fertility of pollen grains. Plant Cell Physiol 51: 896-911

Jin H, Song Z, Nikolau BJ (2012) Reverse genetic characterization of two paralogous acetoacetyl CoA thiolase genes in Arabidopsis reveals their importance in plant growth and development. Plant J 70: 1015-1032

Kasahara H, Hanada A, Kuzuyama T, Takagi M, Kamiya Y, Yamaguchi S (2002) Contribution of the mevalonate and methylerythritol phosphate pathways to the biosynthesis of gibberellins in Arabidopsis. J Biol Chem 277: 45188-45194

Lichtenthaler HK, Schwender J, Disch A, Rohmer M (1997) Biosynthesis of isoprenoids in higher plant chloroplasts proceeds via a mevalonate-independent pathway. FEBS Lett 400: 271-274

Mandel MA, Feldmann KA, Herrera-Estrella L, Rocha-Sosa M, 
Léon P (1996) CLA1, a novel gene required for chloroplast development, is highly conserved in evolution. Plant J 9: 649-658

Nagata N, Suzuki M, Yoshida S, Muranaka T (2002) Mevalonic acid partially restores chloroplast and etioplast development in Arabidopsis lacking the non-mevalonate pathway. Planta 216: 345-350

Newman JD, Chappell J (1999) Isoprenoid biosynthesis in plants: Carbon partitioning within the cytoplasmic pathway. Crit Rev Biochem Mol Biol 34: 95-106

Okada K, Kasahara H, Yamaguchi S, Kawaide H, Kamiya Y, Nojiri $\mathrm{H}$, Yamane H (2008) Genetic evidence for the role of isopentenyl diphosphate isomerases in the mevalonate pathway and plant development in Arabidopsis. Plant Cell Physiol 49: 604-616

Pacini E, Casadoro G (1981) Tapetum plastids of Olea europaea L. Protoplasma 106: 289-296

Phillips MA, D’Auria JC, Gershenzon J, Pichersky E (2008) The Arabidopsis thaliana type I Isopentenyl Diphosphate Isomerases are targeted to multiple subcellular compartments and have overlapping functions in isoprenoid biosynthesis. Plant Cell 20: 677-696

Piffanelli P, Ross JHE, Murphy DJ (1998) Biogenesis and function of the lipidic structures of pollen grains. Sex Plant Reprod 11: 65-80

Preuss D, Lemieux B, Yen G, Davis RW (1993) A conditional sterile mutation eliminates surface components from Arabidopsis pollen and disrupts cell signaling during fertilization. Genes Dev 7: 974-985

Quilichini TD, Douglas CJ, Samuels AL (2014) New views of tapetum ultrastructure and pollen exine development in Arabidopsis thaliana. Ann Bot 114: 1189-1201

Shiba H, Takayama S, Iwano $M$, Shimosato $H$, Funato $M$, Nakagawa T, Che FS, Suzuki G, Watanabe M, Hinata K, et al. (2001) A pollen coat protein, SP11/SCR, determines the pollen $S$-specificity in the self-incompatibility of Brassica species. Plant Physiol 125: 2095-2103

Suzuki M, Kamide Y, Nagata N, Seki H, Ohyama K, Kato H, Masuda K, Sato S, Kato T, Tabata S, et al. (2004) Loss of function of 3-hydroxy-3-methylglutaryl coenzyme A reductase 1 (HMG1) in Arabidopsis leads to dwarfing, early senescence and male sterility, and reduced sterol levels. Plant J 37: 750-761

Suzuki M, Muranaka T (2007) Molecular genetics of plant sterol backbone synthesis. Lipids 42: 47-54

Suzuki M, Nakagawa S, Kamide Y, Kobayashi K, Ohyama K, Hashinokuchi H, Kiuchi R, Saito K, Muranaka T, Nagata N (2009) Complete blockage of the mevalonate pathway results in male gametophyte lethality. J Exp Bot 60: 2055-2064

Villette C, Berna A, Compagnon V, Schaller H (2015) Plant sterol diversity in pollen from Angiosperms. Lipids 50: 749-760 